\title{
Quasi-In-Situ EBSD Observation of the Orientation Evolution in Polycrystalline Tantalum During Rolling Deformation
}

\author{
Ya-Hui Liu' ${ }^{1}$ Shi-Feng Liu ${ }^{1,2} \cdot$ Chao Deng ${ }^{1,2} \cdot$ Hai-Yang Fan ${ }^{3} \cdot$ Qing Liu ${ }^{1}$
}

Received: 17 September 2018 / Revised: 29 November 2018 / Published online: 16 March 2019

(c) The Chinese Society for Metals (CSM) and Springer-Verlag GmbH Germany, part of Springer Nature 2019

\begin{abstract}
The evolution of crystallographic orientation of polycrystalline tantalum (Ta) during rolling was characterized by electron backscatter diffraction technique in a quasi-in-situ way, and the microstructure and microtexture before and after the deformation were characterized and analyzed, respectively. In the specimen, 164 individual grains were exacted singly from the testing region and their corresponding orientations were reconstructed and analyzed, respectively. Results show that the heterogeneous deformation in a grain can be reflected by the accidented surface microstructure. Moreover, the orientations close to $\{111\}$ orientations came closer to the $\{111\}$ corner, while the orientation evolution is more complicated for the orientations close $\{100\}$ corner, indicating that the evolution of these orientations close to $\{100\}$ corner seemed to be irregular.
\end{abstract}

Keywords Tantalum $\cdot$ Orientation $\cdot$ Quasi-in-situ $\cdot$ Rolling

\section{Introduction}

Rolling is a common fabricating technology which was widely used in the processes for strips and sheets of many metals in recent years [1-3]. When compared to other deformation processes like tensile testing or compression, rolling is actually a kind of shear deformation $[4,5]$. During rolling, the shear force is actually a resultant force comprising the downward pressure and forward friction force between the roller and specimen [6]. Rolling deformation leads to the subdivision and rotation of grains, and the rotation of grains leads to the change of orientations. The change of orientations of large amount of grains results in the occurrence of macrotexture of a metal and, in turn, causes the mechanical anisotropy of the metals. [7-9].

Available online at http://link.springer.com/journal/40195

Shi-Feng Liu

liusf06@cqu.edu.cn

1 College of Materials Science and Engineering, Chongqing University, Chongqing 400044, China

2 Electron Microscopy Center of Chongqing University, Chongqing University, Chongqing 400044, China

3 Department of Mechanical Engineering, KU Leuven, Leuven, Belgium
As a metal with body-centered cubic (BCC) structure, tantalum (Ta) has a very high melting point $\left(2996^{\circ} \mathrm{C}\right)$ and density $\left(16.7 \mathrm{~g} / \mathrm{cm}^{3}\right)$, and it is widely used as a kind of sputtering target in the integrated circuit (IC) industry [10]. And uniform texture is vital for tantalum sputtering target to achieve favorable sputtering performance [11]. Thus, the texture should be controllable during the rolling process, and this will be feasible on the premise that there are given laws of orientation evolution during rolling. However, the deformation microstructures and the corresponding characteristics of Ta have not been systematically studied yet, especially the orientation evolution during rolling. The study on the fabricating technique and the subsequent microstructures and texture of metals goes more and more thoroughly with the characteristics technique developing. In particular, the development of electron backscatter diffraction (EBSD) technique provides an excellent way to characterize and analyze the orientation aspects of grains in rolled metals [12-14].

The present study, therefore, investigated the grain orientation evolution during rolling in a quasi-in-situ way to better understand the microstructural characteristics of Ta. To ensure the accuracy of orientation data, the EBSD characterization is mainly focused on the specimen with a low rolling reduction, since the orientations disperse seriously in specimens with medium and large deformation $[9,15,16]$. 


\section{Experimental}

A high-purity Ta sheet with fully recrystallized microstructure was used as a starting material, and the details of chemical components are given in Ref. [17]. A block of $10 \mathrm{~mm} \times 10 \mathrm{~mm} \times 4 \mathrm{~mm}$ specimen was cut off from the Ta sheet, and its TD (transverse direction) plane was carefully polished with sand papers followed by an electropolishing using a mixture of hydrofluoric acid and sulfuric acid (1:9 by volume) at ambient temperature. During the electropolishing process, the specimen served as an anode and the high-purity graphite sheet served as a cathode. The dwell time is about $9 \mathrm{~min}$. The rolling process was carried out on an industrial mill with a roller of $500 \mathrm{~mm}$ in radius. The specimen was rolled uniaxially with one pass, and the total reduction is $17.8 \%$ after the rolling process.

EBSD technique was applied to characterize the microstructure in the middle region of TD plane of the specimen before and after the rolling process, respectively. EBSD measurements were conducted on an accelerating voltage of $20 \mathrm{kV}$ using a JEOL JSM-7800F scanning electron microscope (SEM). The data acquisition and analysis were performed on an Aztec EBSD system (Oxford Instruments) and a HKL Channel 5 software, respectively.

To ensure that the testing plane is clean, the specimen was soaked in acetone for $1 \mathrm{~h}$ followed by a 30-min ultrasonic cleaning at $25^{\circ} \mathrm{C}$ before the EBSD measurement. Besides, during the rolling process, the specimen was first wrapped by a layer of soft papers and the aluminum foil is at the outermost to prevent the pollution.

\section{Results}

Figure $1 \mathrm{a}$ and $\mathrm{b}$ shows the microstructure of the specimen before and after the rolling process, respectively. The rolling, to some extent, can be further divided into stretch deformation along the rolling direction (RD) and compress deformation along the normal direction (ND) [18]. The compress deformation along the ND direction leads to the occurrence of drape-like or concave/convex microstructures in certain regions of the TD plane, and some grains with clear outline emerge after the rolling process. A certain degree of sinking is observed in some grains that have undergone serious deformation, as shown in the left corner of Fig. 1b. Figure $2 a, b$ shows the orientation imaging maps (OIMs) for specimens before and after the rolling process, respectively. The initial grains were elongated along the RD direction and simultaneously compressed along the ND direction (Fig. 2b). Some grains have experienced subdivision as ascertained by the color variation existing in local regions of these grains. Further, the color variations, to some extent, tend to occur in grains with large size. In fact, the color variations reflect the orientation gradients formed in certain regions of a grain.

It should be noted here that several indentation signs were marked on the testing plane of the specimen, and many figures with different magnifications were captured for this testing plane, to ensure that the testing region after rolling is the same one as that before rolling. Furthermore, to minimize the orientation error resulting from the specimen alignment operation, careful position correction work
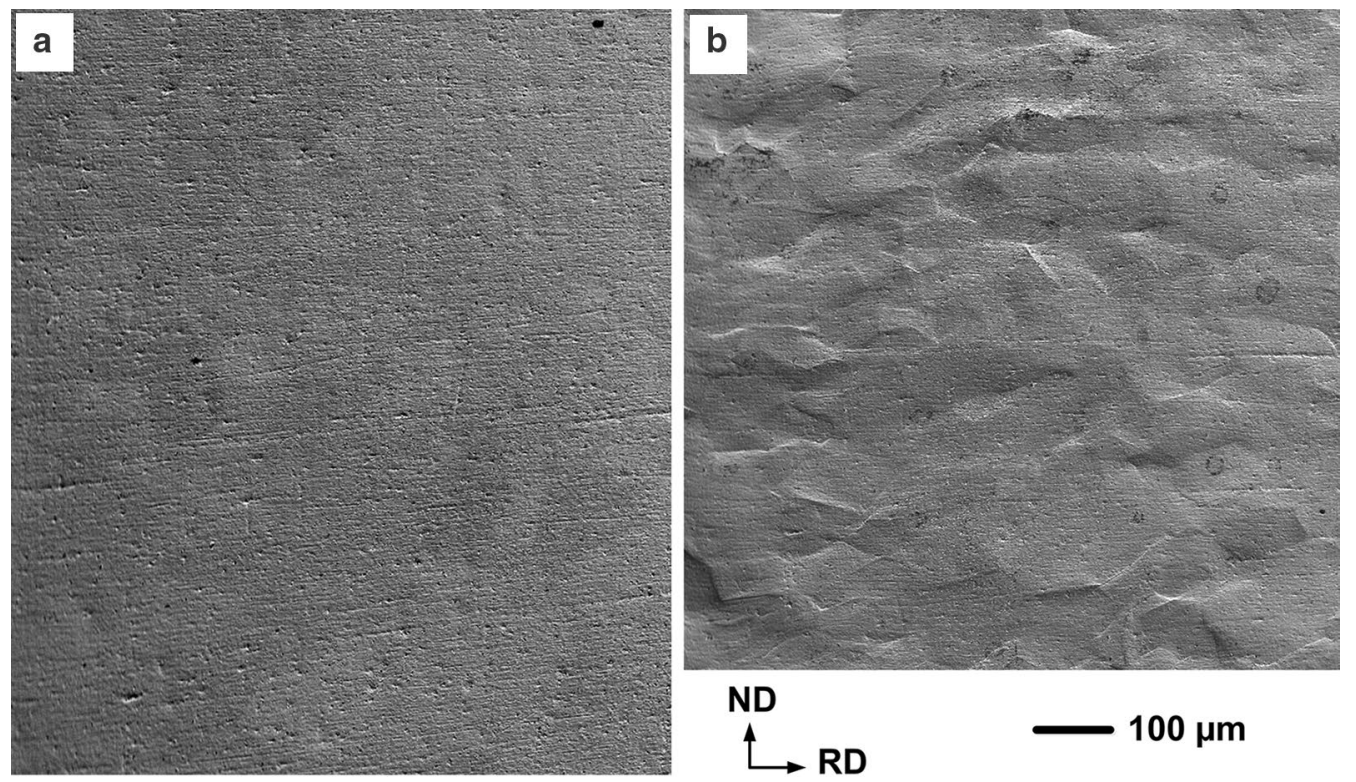

Fig. 1 Scanning electron micrographs of specimens: a before rolling, $\mathbf{b}$ after rolling 

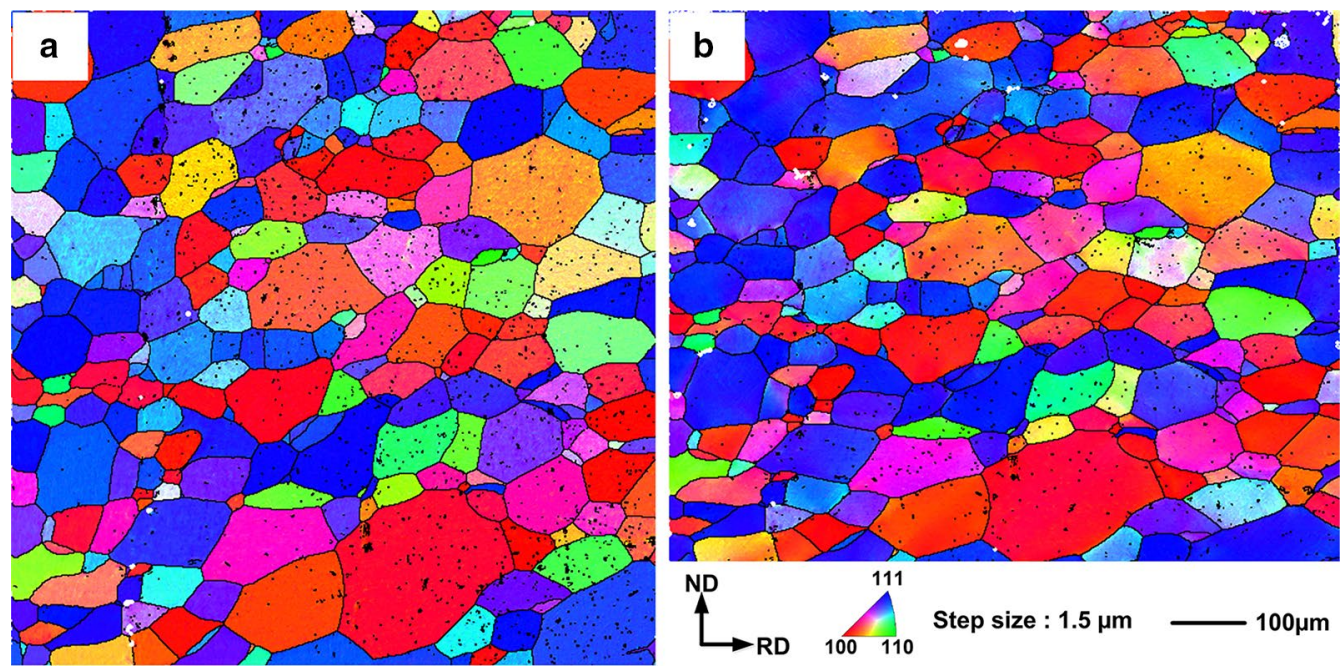

Fig. 2 Orientation imaging maps (OIMs) of specimen: a before rolling, $\mathbf{b}$ after rolling

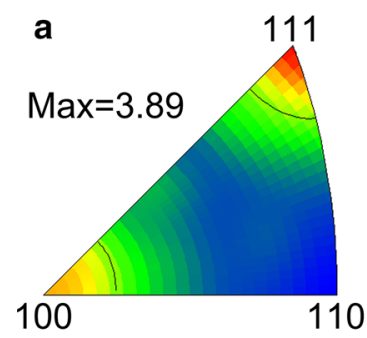

$\{100\}-23.5 \%$

$\{110\}-7.16 \%$

$\{111\}-30.4 \%$

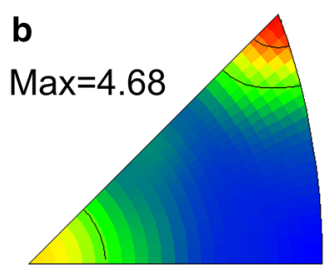

$\{100\}-24.1 \%$

$\{110\}-4.73 \%$

$\{111\}-37.1 \%$

Fig. 3 Inverse pole figures of specimen: $\mathbf{a}$ before rolling, $\mathbf{b}$ after rolling

was carried out on the JEOL JSM-7800F SEM during the measurement of EBSD. We can find that the SEM microstructure (Fig. 1) and EBSD OIMs (Fig. 2) before and after the rolling are in good agreement in this investigation.

Figure $3 \mathrm{a}$ and $\mathrm{b}$ shows the inverse pole figures (IPFs) of the specimen before and after rolling, respectively. As shown in the IPFs, the maximal orientation density increases from 3.89 to 4.68 after rolling deformation. In particular, an orientation concentration occurred in $\{111\}<u v w>(<111>/ / \mathrm{ND})$ microtexture, and the $\{111\}<$ uvw $>$ texture increases from $30.1 \%$ to $37.1 \%$, while the content of $\{110\}<$ uvw $>(<110>/ / N D)$ decreases from $7.16 \%$ to $4.73 \%$. The volume fraction of $\{100\}<u v w\rangle(<100>/ / \mathrm{ND})$ texture seems to be basically unchanged after the rolling process.
It is necessary to systematically investigate the orientation evolution of a single grain during rolling for establishing the relationship between the macrotexture and the deformation methods, since the change of macrotexture is essentially due to the changes of large amounts of single grain orientations in specimen. Figure 4 shows the grain boundary maps before and after the rolling process, and these grains are numbered successively through the thickness of the testing region. Note that there is a one-to-one correspondence between the grain numbers before and after rolling. In total, 164 grains were investigated in this study, as shown in Fig. 4. Then, their orientations and the corresponding points in IPF before and after rolling were exacted and marked by Channel 5 software, respectively. As shown in Fig. 5, the dot represented the initial grain orientation, and the line linked the initial and final grain orientations. The evolution of the orientations presents a significant trend related to the initial orientations. In other words, the orientation evolution is orientation dependent.

According to the orientation evolution paths, the IPF can be further divided into four parts, as shown in Fig. 5. Accurately, orientations in region 1 rotate toward the $\{100\}$ corner, but the orientations become dispersed when they are close to the $\{100\}$ corner. Orientations in region 2 are close to the $\{110\}$ corner before rolling, and they rotate toward the $\{111\}-\{100\}$ line in IPF after rolling. Orientations in region 3 are close to the $\{112\}$ and $\{111\}$ orientations before rolling, while they become dispersed after rolling, and some of them rotate toward the $\{111\}$ corner. Then, orientations in region 4 are close to $\{221\}$ before rolling, and they rotate toward the $\{111\}$ corner after rolling. 


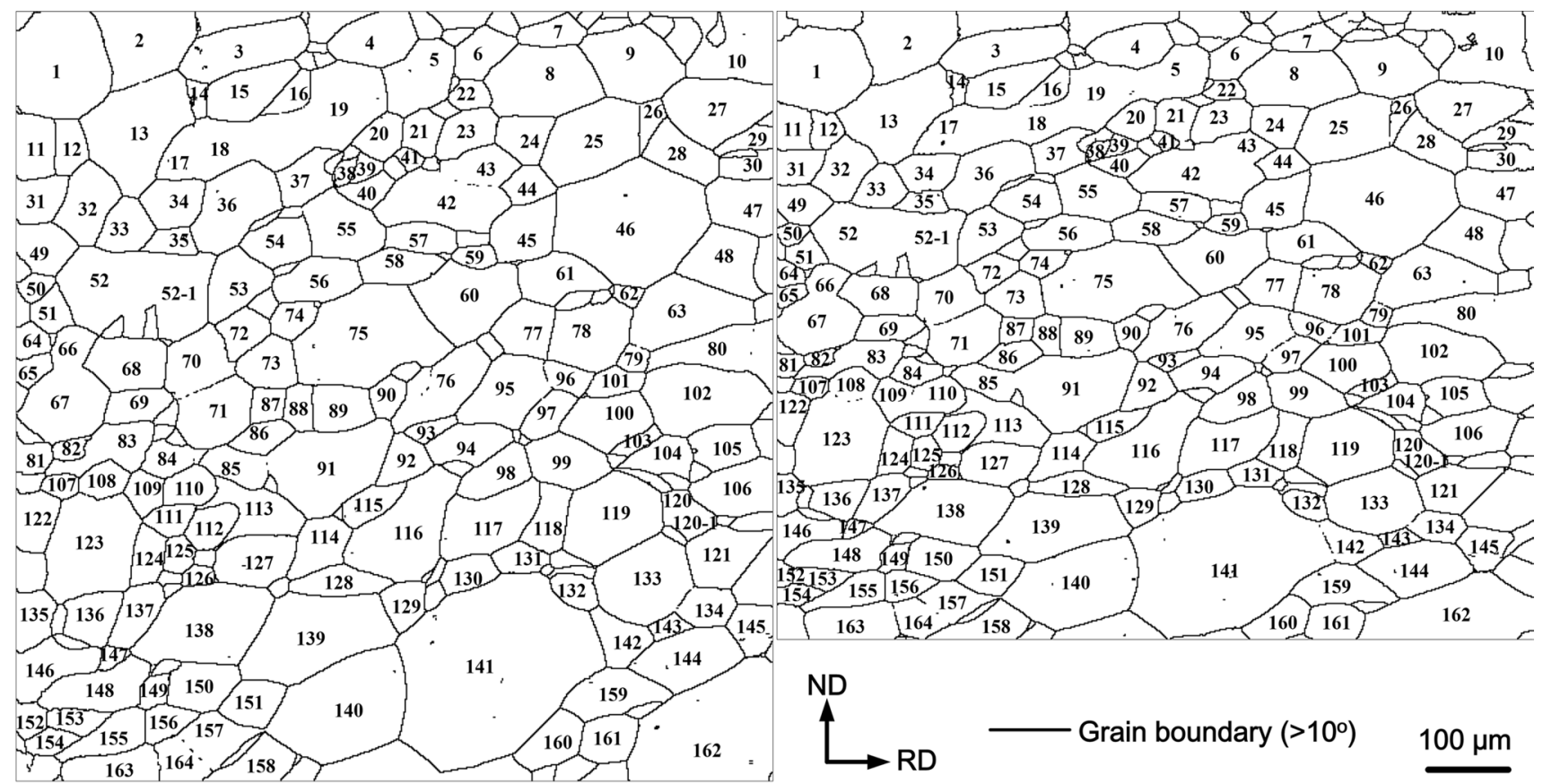

Fig. 4 Grain boundary maps of specimen before and after rolling. Grains are numbered successively through the thickness

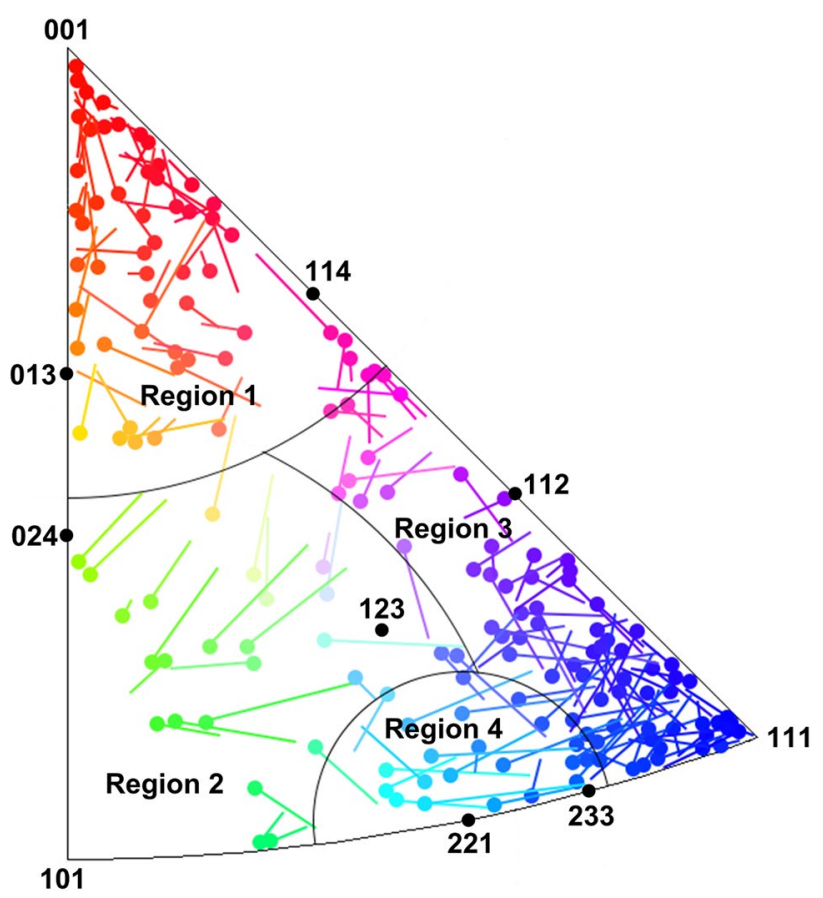

Fig. 5 Grain orientations before and after rolling. The dot represents the initial grain orientation, and the line linked the initial and final grain orientation

\section{Discussion}

During the rolling deformation, a certain plane slips along a specified direction, and these certain plane and direction can be termed as a slip system. The deformation of many metals, especially those with BCC structure, is always realized by activating these slip systems in grains [19-21]. In fact, the face-centered cubic (FCC) metals always deform based on the $\{111\}<110>$ slip systems, and the BCC metals may deform based on the $\{110\}<111>,\{112\}<111>$ or $\{123\}<111>$ slip systems [20,22]. The ductility is relatively poor in hexagonal close-packed (HCP) metals due to their finite slip systems [23]. The grains are closely packed in these polycrystalline metals, and the grain boundaries are closely connected with each other. The activation of certain slip systems is not an isolated occurrence, and it is usually associated with the rotation of crystal lattice to guarantee that the adjacent grains are closely packed after the deformation. Thus, the activation of slip systems accompanied with the rotation of the crystal lattice leads to the change of orientations [2, 24, 25]. Besides, the activation of slip systems is not uniform in one grain, and different slip systems may be activated in different regions in the same grain [26]. Then, the drape-like microstructure occurs on the specimen surface during the deformation. And the more serious the deformation of the grain experienced, the more the drapelike microstructures [27].

As shown in Fig. 1, some drape-like microstructures appear on the TD plane of specimen, so the TD plane looks rugged 
after the rolling deformation. Different regions presented different degrees of concave/convex in this plane, or even the degrees are not uniform in the same grain [25]. As shown in the OIM in Fig. 2b, orientation gradients or orientation dispersion occurs in some grains after rolling, which also implies that the deformation is not homogeneous in a grain. This heterogeneous deformation mainly results from the situation that different slip systems are activated in different regions during rolling [28]. And this is in a good agreement with the deformation of austenitic stainless steel upon equal-channel angular pressing [25]. As reported, $\{111\}$ and $\{100\}$ orientations are the two main textures in deformed Ta [16]. Most orientations rotate toward the $\{111\}$ or $\{100\}$ corner after rolling in the present investigation, and the initial grains near the $\{111\}$ or $\{100\}$ corner remain being around the corresponding orientation type after rolling, indicating that $\{111\}$ and $\{100\}$ orientations are relatively stable. As compared to the two orientations, grains close to $\{110\},\{122\}$ and $\{123\}$ orientations rotate toward the $\{111\}$ or $\{100\}$ corners during rolling, indicating that they are unstable orientations.

Although all grains rotate for certain degrees during rolling, the rotation degrees in different grains are not the same [29]. The rotation degree is also orientation dependent to some extent. According to our previous study, the deformation of certain regions in $\{111\}$ gains can be maintained by the operation of a single slip system, the primary slip system, and the rotation of $\{111\}$ grain adhered to a certain set of rules or restrictions [6]. As shown in Fig. 5, the initial orientations near the $\{111\}$ orientations before rolling tend toward the $\{111\}$ orientations after rolling. But the rotation behaviors are very complicated for $\{100\}$ oriented grains, since these grains have a tendency to activate multiple slip systems to coordinate the deformation [30]. Thus, the orientations come to be dispersed when they are close to $\{100\}$ orientations. But the degree of dispersion is not large in this study, which means that the rotation angle of a grain is limited during rolling. This mainly results from the deformation mechanism of $\{100\}$ orientations. $\{110\}<111>$ and $\{112\}<111>$ slip systems are often activated in BCC metals at low/mid-temperatures, and these two main slip systems can be further divided into 24 pairs of slip systems [31]. This implies that the rotation angle between adjacent slip systems is very small, indicating that the degree of the orientation change is limited during deformation. Therefore, the initial orientations near $\{100\}$ before rolling kept being around $\{100\}$ after rolling.

\section{Conclusions}

EBSD was used for quasi-in-situ characterization of the orientation evolution of tantalum during rolling in this study, the microstructures of the specimen before and after the rolling were analyzed, and the main conclusions are as follows.

1. Some concave/convex microstructures appeared on the TD plane after rolling, and the distribution of these microstructures is not homogeneous, indicating the heterogeneous deformation of Ta.

2. The evolution of orientations of 164 individual grains indicated that the change of orientation and the rotation angle of orientation are orientation-dependent.

3. The initial orientation near $\{111\}$ before rolling rotates toward the $\{111\}$ corner, but after rolling, the orientations come to be dispersed for $\{100\}$ orientations. This mainly results from the single slip system operated in $\{111\}$ grains and the multiple slip systems operated in $\{100\}$ grains.

Acknowledgements The present work was co-supported by the National Natural Science Foundation of China (Nos. 51421001 and 51701032), the Major National Science and Technology Projects of China (No. 2011ZX02705) and the Chongqing Science and Technology Commission in China (CSTC, 2017jcyjAX0094). We are deeply grateful to Dr. Qianqian Zhu's help in the grammar polishing of this manuscript.

\section{References}

[1] C.X. He, F.Y. Yang, G. Ma, X. Chen, L. Meng, Acta Metall. Sin. (Engl. Lett.) 29, 554 (2016)

[2] J.Y. Xia, L.J. Chai, H. Wu, Y. Zhi, Y.N. Gou, W.J. Huang, N. Guo, Acta Metall. Sin. (Engl. Lett.) 31, 1215 (2018)

[3] Y.Q. Zhao, H.M. Chen, J. Zhang, R. Ma, Y.D. Liu, Y.N. Wang, L. Wang, Q. Zhang, W.G. Li, Acta Metall. Sin. (Engl. Lett.) 31, 673 (2018)

[4] Z. Yanushkevich, A. Belyakov, R. Kaibyshev, C. Haase, D.A. Molodov, Mater. Charact. 112, 180 (2016)

[5] T.R. Li, G.H. Liu, M. Xu, T.L. Fu, Y. Tian, R.J.D.K. Misra, Z.D Wang, Acta Metall. Sin. (Engl. Lett.) 31, 933 (2018)

[6] Y.H. Liu, S.F. Liu, J.L. Zhu, C. Deng, H.Y. Fan, L.F. Cao, Q. Liu, Mater. Sci. Eng. A 707, 518 (2017)

[7] L. Sun, K. Muszka, B.P. Wynne, E.J. Palmiere, Scr. Mater. 64, 280 (2011)

[8] H. Paul, J.H. Driver, C. Maurice, M. Buak, Arch. Metall. Mater. 51, 43 (2006)

[9] B.L. Li, A. Godfrey, Q. Liu, Scr. Mater. 50, 879 (2004)

[10] S.M. Cardonne, P.K.C.A. Michaluk, H.D. Schwartz, Int. J. Refract. Metals Hard Mater. 13, 187 (1995)

[11] C.A. Michaluk, J. Electron. Mater. 31, 2 (2002)

[12] S.I. Wright, M.M. Nowell, S.P. Lindeman, P.P. Camus, M. De Graef, M.A. Jackson, Ultramicroscopy 159, 81 (2015)

[13] N.C.K. Lassen, D.J. Jensen, Acta Cryst. 50, 741 (1994)

[14] A.J. Wilkinson, T.B. Britton, Mater. Today 15, 366 (2012)

[15] K. Hagihara, M. Yamasaki, M. Honnami, H. Izuno, M. Tane, T. Nakano, Y. Kawamura, Philos. Mag. 95, 132 (2014)

[16] H. Fan, S. Liu, L. Li, C. Deng, Q. Liu, Mater. Des. 97, 464 (2016)

[17] C. Deng, S.F. Liu, X.B. Hao, J.L. Ji, Z.Q. Zhang, Q. Liu, Int. J. Refract. Metals Hard Mater. 46, 24 (2014)

[18] J.R. Luo, A. Godfrey, W. Liu, Q. Liu, Acta Mater. 60, 1986 (2012) 
[19] K. Kashihara, T. Shibayanagi, Mater. Trans. 50, 2192 (2009)

[20] G. Winther, L. Margulies, S. Schmidt, H.F. Poulsen, Acta Mater. 52, 2863 (2004)

[21] D. Raabe, Mater. Sci. Eng. A 186, L1 (1994)

[22] I.L. Dillamore, W.T. Roberts, Acta Meter. 12, 281 (1964)

[23] T.Z. Han, G.S. Huang, L. Huang, B. Jiang, G.G. Wang, A.T. Tang, F.S. Pan, Acta Metall. Sin. (Engl. Lett.) 31, 225 (2017)

[24] Y.L. Wei, A. Godfrey, W. Liu, Q. Liu, X. Huang, N. Hansen, G. Winther, Scr. Mater. 65, 355 (2011)

[25] F.Y. Dong, P. Zhang, J.C. Pang, Q.Q. Duan, Y.B. Ren, K. Yang, Z.F. Zhang, Acta Metall. Sin. (Engl. Lett.) 29, 140 (2016)

[26] X. Jin, B.Q. Fu, C.L. Zhang, W. Liu, Acta Metall. Sin. (Engl. Lett.) 28, 1149 (2015)
[27] X. Huang, N. Tsuji, N. Hansen, Y. Minamino, Mater. Sci. Eng. A 340, 265 (2003)

[28] Q.Z. Chen, A.H.W. Ngan, B.J. Duggan, Proc. R. Soc. A Math. Phys. Eng. Sci. 459, 1661 (2003)

[29] L. Liu, J. Meng, J.L. Liu, H.F. Zhang, X.D. Sun, Y.Z. Zhou, Acta Metall. Sin. (Engl. Lett.) 32, 831 (2019)

[30] H. Wang, C.J. Boehlert, Q.D. Wang, D.D. Yin, W.J. Ding, Int. J. Plast 84, 255 (2016)

[31] Y. Liu, S. Liu, J. Zhu, H. Fan, C. Deng, L. Cao, X. Wu, Q. Liu, J. Mater. Sci. 53, 12543 (2018) 